\title{
The Effect of Object Color Tones on Emotions
}

\author{
Zimeng Chen ${ }^{1, \dagger}$, Xiangrong Meng ${ }^{2, *, \dagger}$, Zhiyu $\mathrm{Yao}^{3, \dagger}$
}

\author{
${ }^{1}$ China Agriculture University,Beijing, China \\ ${ }^{2}$ Jiangxi University of Chinese Medicine, Jiangxi, China \\ ${ }^{3}$ Zhejiang University, Zhejiang, China \\ ${ }^{*}$ Corresponding author. Email: 201801024011@jxutcm.edu.cn \\ These authors contributed equally.
}

\begin{abstract}
The effect of color on emotions has always been significant in many fields such as advertising and communication. In order to verify the influence of colors on emotions, this study divides colors into warm and cold tones, and emotions are divided into positive emotions and negative emotions. In this study, a program written by PsychoPy was used to show the participants four pictures. This study collected the emotional scores of the participants after viewing the pictures and compared and analyzed the influence of the two tones on different emotions. The experimental results show that when the content of the experiment is red or yellow, the participants are more likely to have positive emotions, and when the content of the experiment is blue or green, they are more likely to have negative emotions.
\end{abstract}

Keywords: Color tone, Emotion, Cool-tone, Warm-tone.

\section{INTRODUCTION}

Emotional health plays a significant role in people's quality of life. The research of psychological immunology shows that optimism may be related to the improvement of immune function [1], and people often receive environmental stimuli from all aspects of their daily life, which will always affect people's emotional health to varying degrees. Among these stimuli, color is often an easily overlooked point. The direct impact of color on people's emotional health comes from the physical light stimulation of color on people's physiology. Hue, saturation, and brightness are the three perceptual dimensions of the color [2]. Psychologists have found that in the red environment, people's pulse will speed up, their blood pressure will rise, their emotions will be excited, impulsive, and aggressive; in the blue environment, the pulse will slow down, and the mood is relatively calm; and green makes people relaxed and shows fatigue and guilt [3]. In addition to the direct psychological effect of color, color also has a more complex indirect psychological effect. At this time, the psychological effects caused by color not only stay at the superficial level of perception or perception but lead to more profound psychological activity processes, such as association, thinking, and even memory [4]. Using color to improve people's emotional health has gradually become a more common treatment.
It is reported that the apparent effect of pink on calming emotions has been observed in the naval detention centre in Seattle, the youth home in San Bernardino, California, the psychiatric ward of Los Angeles Veterans Hospital, and the South Bronx school for hyperactive children [5].

Color is highly affected by regionality. For example, in China, red is a festive color. Most Chinese people's emotions unconsciously turn to joy at the sight of red. But in Thailand, red is an unlucky color. In different cultures, different colors have very different effects on people's emotional health. Only by fully understanding the appreciation habits and aesthetic psychology of different objects, mastering the psychological law of people's understanding and appreciation of color, and using color reasonably can people maintain good emotional health [6]. The effect of color on human mood has been revealed, but supporting data is still scarce. Therefore, further experiments are necessary.

This study aims to test the effect of color on emotion. We assume that hue may cause psychological mood changes. Our experiment uses the PsychoPy program to investigate whether different color hues will affect psychological emotion and tries to study the phenomenon and possible mechanism of the influence of color on emotion through cognitive experimental design. 


\section{METHODS}

\subsection{Participants}

Twenty volunteer participants were recruited at three universities in China. All participants were between the ages of 18 and 24(mean age 20). They had to be identified in advance for psychological and eye disorders that could affect color recognition.

Every time they finished an image watch, they were required to score the four emotions according to the chart.

This scale consists of a number of words that describe different feelings and emotions. Remand each item and then list the number from the scale below next to each word. Indicate to what extend you feel the way right now, that is, at the present moment or indicate the extend you have felt this way in the whole experiment.

\subsection{Experimental materials}

Our experiment measured hues including red, yellow, blue, and green. The blank control group in our experiment is a colorless picture. The experimental color saturation is $78-85 \%$ and luminosity is $62 \%$. We measured four emotions: distressed, upset, excited, and interested.

The Natural Color System uses six colors as solid or primary colors: white, black, yellow, red, blue, and green. White and black are non-color, and the other four are colors.

In order to clearly distinguish the difference between different color groups and to make the experiment specific and easier to operate, yellow, red, blue, and green were chosen as the experimental colors.

In order to control the saturation and lightness, fourcolor images were drawn by Procreate (Savage Interactive Pty Ltd, 2011). PsychoPy was used to write the program. To make sure all participants see the same image, the equipment used in the experiment was a computer of the same model, with a screen resolution of $1400 \times 900$ and a size of 15 inches.

Positive and Negative Affect Schedule (PANAS) was chosen to be applied to this experiment as a quantitative measure of the participants' emotions [7]. However, considering the patience of the participants and time cost, also for the convenience of data collection, we only selected four of the twenty emotions in the original scale. In order to maintain balance, we chose two positive emotions (excited and interested) and two negative emotions (distressed and upset).

\subsection{Experimental procedure}

Before the experiment began, we obtained informed consent from the participants and they were told that the experiment would not cause any physical or mental damage to them.

The laboratory lighting environment is fluorescent lamp, a fluorescent lamp for ambient light is conducive to the fidelity of the color, and the color rendering is not affected by natural light during the day and night. The cool white bulb may bring some changes to the color. Participants sat with their backs to the window to control other light sources from the window not to interfere with the screen color too much. Participants were arranged to sit at a table in the laboratory, and the computer was placed on the table. The participant could pick up the computer or place it on the table during the experiment.

Participants were asked to watch five images that are identical except for the color, and each image lasted ten seconds in the center of the screen. While the environment was quiet, their mood was relaxed. The images were shown in colorless, red, yellow, blue and green. These images were presented in the same order.

Each time they finished viewing an image, a text instruction appeared on the screen and participants were asked to self-assess themselves on a scale of ' 1 ' to ' 5 ' for each of four emotions.

\section{RESULTS}

Descriptive statistics of baseline trial were made on the scores of color's influences on psychological mood, by using the descriptive analysis function in SPSS.

The final data is integrated into a bar graph, in order to confirm whether there are significant differences in the data between the groups, and for further analysis, we performed the analysis of variance on the data (On the horizontal axis in all bar charts, ' 0 ' means colorless, ' 1 ' means red, '2' means yellow, ' 3 ' means blue, ' 4 ' means green).

The 'sig' value of each emotion score is less than 0.05 , so each group of data has significant differences, which can prove that each color has a significant impact on different emotions.

Figure 1 documented the average score of upset mood of participants in the five experimental colors, with error bars showing one standard deviation from the mean. The stimulation level of red and yellow to 'upset' is lower than that of the blank control group, while blue and green are higher. The blue group has the highest score, and the red group has the lowest score. 


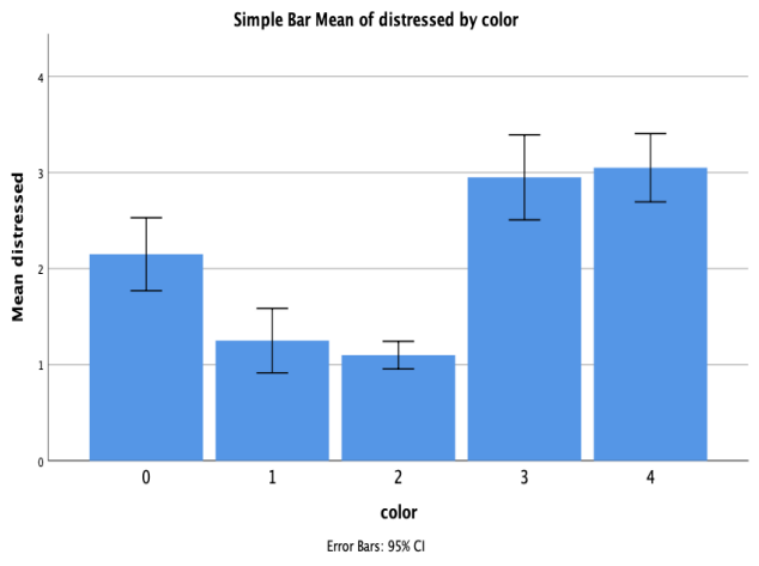

Figure 1. Simple bar mean of upset by color

As shown in figure 2 below, the stimulation level of red and yellow to 'distressed' is lower than that of the blank control group, while blue and green are higher than the control group. The green group has the highest score and the yellow group has the lowest score.

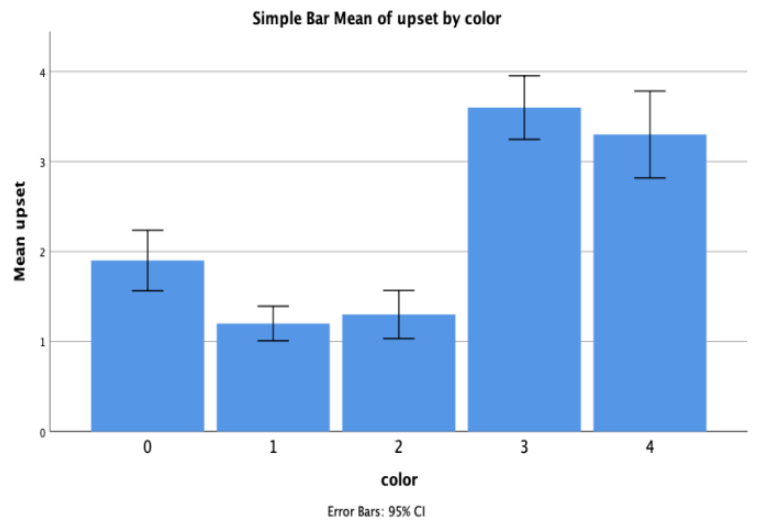

Figure 2. Simple bar mean of distressed by color

As shown in figure 3 , the stimulation levels of the four colors of red, yellow, blue, and green on 'excited' are higher than those of the blank control group, but the stimulation levels of red and yellow are significantly higher than those of blue and green. The red group has the highest score, and the green group has the lowest score.

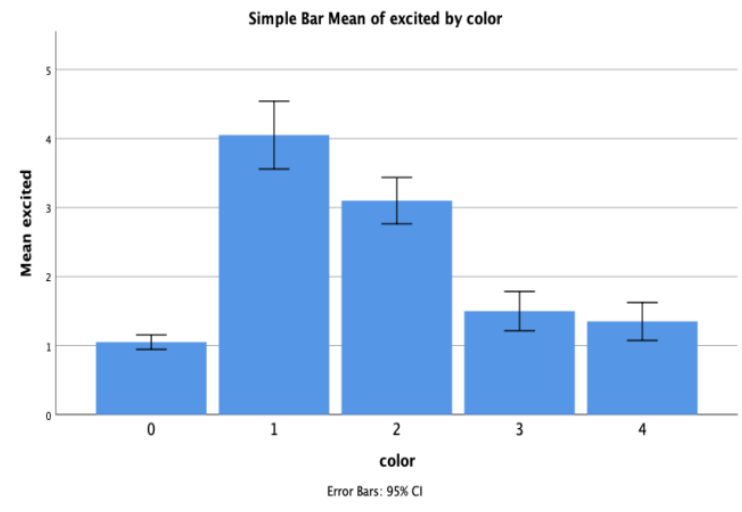

Figure 3. Simple bar mean of excited by color
According to figure 4 below, the stimulation levels of the four colors of red, yellow, blue, and green on 'interested' are higher than those of the blank control group, but the stimulation levels of red and yellow are significantly higher than those of blue and green. The red group has the highest score and the blue group has the lowest number.

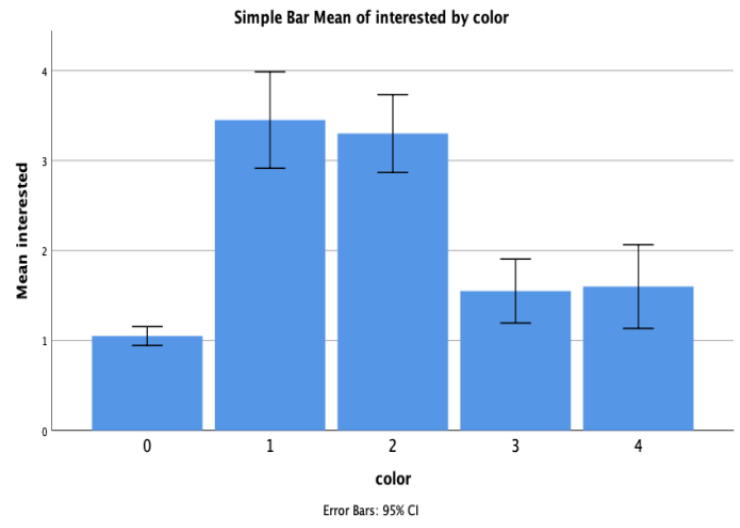

Figure 4. Simple bar mean of interested by color

The visual color of an image can affect a person's mood. Warm colors like red and yellow are more likely to lead to positive emotions and cool colors like blue and green are more likely to lead to negative emotions.

\section{DISCUSSION}

Our experiments demonstrated that participants were more prone to produce positive emotions (such as 'interested', 'excited') when the experimental content was in red or yellow, and they were more likely to produce negative emotions (such as 'distressed', 'upset', 'alert') when the experimental content was blue or green.

The result of this study supports that color tone could lead to psychological mood variation. For example, the red tone evokes slight excitement and stimulation on psychological mood, the blue tone evokes calmness and quietness. It also explains the hidden reason of symbolic function of different colors on expressing or signaling a certain psychological mood, used in everyday life, such as blue lights right next to railways in Japan to reduce suicidal behaviors. Some studies have found that people's pulse rate will increase in warm colors, blood pressure will rise slightly, and mood will easily rise along; while in cool colors, pulse rate would slow down with psychological mood getting less excited [8]. The result is consistent with our experimental findings, both concluding that emotions get excited (positive) in the warm color-toned environment and low (negative) in the cool color-toned environment.

The explanation for the above phenomena could be the visual stimulation of colors. The associations of life 
experience and environmental things are generated in the thinking aspect, which is the psychological feeling perceived from colors. Red, yellow, orange, and other hues give people a strong visual stimulus, which reminds people of the warm sun and fire, and they perceive relevant information of warmth, so these colors are called warm colors [9]. Another explanation of our experimental results is that, due to the joint perception of our life experience, when we see warm color content, we associate some warm things and then produce similar positive emotions, but when we see cool-tone content associate some cold things, we produce similar calm and quiet emotions [10].

Our experiment tested the emotional response scores of participants to pictures of different tones. However, our experimental results were limited by the sample size, small experimental samples lead to the increasing of random errors, and decreases statistically significant. Moreover, we found that break time was not schedule for the participants throughout the experiment, considering the 10to20-minute length of the complete procedure, which could have led to fatigue effects. Fatigue effect refers to that, due to the long experiment time, the participants are prone to mood swings such as irritability and boredom in the later stage of the experiment. Another limitation is habituation error, which means that participants were accustomed to the sensation caused by the original color stimulus, and still reported the original sensation when the stimuli and sensation had already changed. For improving the experiment, we suggest increasing the sample size, the types of experimental color, and adjust break time during the experiment. Also, we will conduct more indepth research on this experiment, for example: by experimenting with the combination of color and picture content (green and tea, refrigerator and white) to explore whether the matching degree of a picture color and the content will affect the mood of the audience.

Based on the above research, it is meaningful to conduct further experiments in the direction of color and picture content. Color can make people associate and feel. In the design of advertising pictures, using the law of color and emotion can better express the advertising theme, arouse people's emotions, arouse people's interest in advertising and advertising products, and ultimately affect people's choices. Our experiment has great reference significance and application value for how to effectively improve consumers' brand perception value through the scientific selection of advertising colors. The experimental results also remind marketers that they should be fully aware of the important role of color in advertising pictures, and make full use of the role of advertising color collocation to promote consumer brand perception value.

\section{CONCLUSION}

In summary, we found a statistically significant association between the tone of the experimental content and the psychological mood of the participants. Participants were more prone to produce positive emotions when the experimental content was in red or yellow, and they were more likely to produce negative emotions when the experimental content was blue or green.

\section{REFERENCES}

[1] Kamen-Siegel. L, Rodin. J, Seligman. M. E, \& Dwyer. J, Explanatory style and cell-mediated immunity in elderly men and women. Health psychology: official journal of the Division of Health Psychology, American Psychological Association, 10(4) (2009) 229-235.

[2] Wilms. L \& Oberfeld. D. Color and emotion: effects of hue, saturation, and brightness. Psychological Research, 82 (2018) 896-914.

[3] Schmalzing. D, Tsao. N, Koutny. L, Chisholm. D, Srivastava. A, Adourian. A, Linton. L, McEwan. P, Matsudaira. P \& Ehrlich. D, Toward real-world sequencing by microdevice electrophoresis. Genome research, 9(9) (1999) 853-858.

[4] He. R, The effect of color on emotions. Art Panorama, 1 (2009) 72-73.

[5] He. M, Analysis of the effect of color on people.Decoration Refurbishment Chntre, 6 (2017) 95.

[6] Yang. N. L, Research on the Effect of Color on People's Psychological Feeling.The Science Education Article Collects, 6 (2006) 169-170.

[7] Watson. D, Clark. L. A \& Tellegen. A, Development and validation of brief measures of positive and negative affect: The PANAS scales. Journal of personality and social psychology, 54(6) (1988) 1063.

[8] Hou. M \& Ren. L. Q, The effect of color matching on consumer brand perception value in advertising pictures. Proceedings of 2019 International Conference on Management Innovation, Education Reform and Applied Social Science (MIERASS 2019).

[9] Arbib. M. A, From grasp to language: Embodied concepts and the challenge of abstraction. Journal of Physiology-Paris, 102(1-3) (2008) 4-20.

[10] Avery. N. G, Alan. J. F \& Laurie. A. L, The color of emotion: A metric for implicit color associations. Food Quality and Preference, 52 (2016) 203-210. 